\title{
LA PROFONDEUR DES DISPOSITIFS CIVILISATEURS : LA DIALECTIQUE NÉGATIVE ENTRE LA POLITESSE ET LA MORALITÉ CHEZ L'ANTHROPOLOGIE DE KANT
}

\author{
THE DEPTH OF CIVILIZING FEATURES: \\ NEGATIVE DIALECTICS BETWEEN POLITENESS \\ AND MORALITY IN KANT'S ANTHROPOLOGY
}

\author{
NURIA SÁNCHEZ MADRID* \\ $U C M$
}

RÉsumé: Cet article analyse la contribution que la belle apparence de la vertu, dont la multiplicité de formes peut se réunir autour le nom générique de decorum, offre au procès de civilisation et aussi à la progressive moralisation de l'espèce humaine selon l'anthropologie morale de Kant. Premièrement, je m'arrêterai sur les vertus nommées homilétiques chez la Métaphysique des moeurs, c'est-à-dire, sur la loquacité, la politesse, l'hospitalité et l'indulgence, qui forment partie du effet de trompe d'œil que le sujet pratique doit produire sur sa même nature, encline à l'égoïsme et à la perversion des moteurs morales. En deuxième lieu, j'analyserai le lien entre ces «suppléments de la vertu " qui sont les bonnes manières sociales, et un fait anthropologique incontestable,

* L'auteur souhaite remercier les Projets Poetics of Selfhood: memory, imagination and narrativity (PTDC/MHC-FIL/4203/2012), octroyé par le FCT du Gouvernement de Portugal, et les Projets Naturaleza humana y comunidad (III). ¿Actualidad del humanismo e inactualidad del hombre? (FFI2013-46815-P) et Retóricas del Clasicismo. Los puntos de vista (contextos, premisas, mentalidades) (FFI2013-41410-P), octroyés par le MINECO du Gouvernement de l'Espagne, pour leur support dans l'élaboration de cet article. Une version réduite et préalable du travail a été lue au XIème Congrès de la SEKLF de Salvador de Bahia, en octobre de 2013. Je remercie vivement les collègues Michèle Cohen-Halimi (Univ. Paris-Ouest) et Sarah Martín (UEM) pour leur conseils linguistiques sur mon texte. 
c'est-à-dire la faiblesse de la nature humaine et son besoin d'user des béquilles esthétiques afin de s'accoutumer à agir suivant des principes pratiques pures. Finalement, je parlerai de l'importance de la remarque kantienne qui soutient que les parerga de la vertu doivent être cultivés dans le milieu de la vie sociale, c'est-à-dire toujours en contact avec les autres. Par le contraire, l'apparence morale produite par le sujet individuel sera interdite sans ambages, parce que chacun doit éviter tomber dans faux illusions sur la pureté de ses intentions.

MoTS CLÉ: Kant, politesse, civilisation, culture, moralisation, decorum

AвSTRACT: This paper tackles the contribution that the beautiful appearance of virtue, whose multiplicity of forms can be gathered under the generic name of decorum, offers to the civilizing process and also to the progressive moralization of mankind according to Kant's Moral Anthropology. First, I will focus on the homiletic virtues analyzed in the Metaphysics of Morals, that is, loquacity, politeness, hospitality and indulgence, which bring about a trompe l'oeil inside the subject, always prone to selfishness and to the perversion of its practical drives. Secondly, I will analyze the relationship between these "supplements of virtue," good social manners, and an undeniable anthropological fact, that is, the weakness of human nature and the need to use aesthetic crutches to get accustomed to acting in accordance with pure practical principles. Finally, I will cast light on the importance that parerga deserves according to Kant, who argues that these special virtues must be cultivated in the midst of social life, that is to say, always in contact with others. On the contrary, moral appearance produced by the individual subject ought to be banned unequivocally, because everyone must avoid falling into false illusions about the purity of her or his own intentions.

KeYwords: Kant, Politeness, Civilization, Culture, Moralization, decorum.

J'aimerais commencer par la formulation kantienne d'une situation embarrassante, qui met en évidence que les efforts encouragés par les savants afin que les hommes s'approchent fraternellement les uns des autres n'apportent pas souvent les résultats espérés :

"Un auteur demande à un de ses lecteurs : comment trouvez-vous mon œuvre ? On pourrait bien donner une réponse illusoire en se moquant de ce qu'une telle question a d'embarrassant, mais qui a toujours prêt un mot 
d'esprit ? La moindre hésitation à répondre est déjà une humiliation pour l'auteur, le lecteur peut-il lui parler selon son cœur ?». ${ }^{1}$

Le texte laisse supposer que le Witz — le mot d'esprit_-, bienveillant pour la nature humaine, serait souvent de meilleure compagnie que la franchise, qui ne ferait que blesser l'interlocuteur. La correspondance de Kant est pleine d'exemples dans lesquels notre auteur cherche à s'excuser d'actes susceptibles d'éveiller légitimement l'indignation d'autrui. Retards de livraison des textes promis aux éditeurs, délais de réponse aux correspondants parce qu'un calcul des priorités impose qu'on consacre davantage de temps au travail académique... dans tous ces cas, le sujet de l'énonciation se présente face à l'autre comme un sujet labile, plein de failles, mais bien conscient des tâches qu'il doit encore entreprendre pour atteindre le but de sa réussite comme sujet moral, c'est-à-dire comme personne. L'image d'un Kant paladin du rigorisme moral est bien connue, mais ses remarques sur la convenance qu'il y a à éduquer les jeunes selon les règles de la politesse restent plus ou moins évidentes pour les interprètes. Il admet en outre qu'écrire dans une lettre «je suis votre très obéissant serviteur» ${ }^{2}$ est un acte qu'on ne peut pas condamner parce qu'il ne trompe personne ; bien entendu le sens commun règne toujours dans une société. Pour qui est encore dénué d'une véritable vertu l'imitation d'une conduite morale peut servir de préparation esthétique favorisant l'adoption de la forme d'agir morale. Cette virtualité permet de considérer les comportements tels que l'affabilité et la sociabilité comme des suppléments anthropologiques de la moralité rapprochant les hommes de la vertu pour ainsi dire du point de vue de l'extériorité, dans l'attente que la souhaitable et nécessaire révolution du cœur se produise dans leur intériorité.

Dans cette communication, je me propose d'analyser et de délimiter la contribution que la belle apparence de la vertu, dont la multiplicité de formes peut se réunir autour du nom générique de decorum, offre au procès de civilisation et aussi à la progressive moralisation de l'espèce humaine, selon le point de vue de l'anthropologie morale de Kant. Mon argumentation s'articulera en trois parties. Premièrement, je m’arrêterai sur les vertus nommées homilétiques dans

${ }^{1} M M, D V$, AA 06: 431. Le références aux œuvres de Kant suivent l'édition et la pagination de l'Académie des Sciences de Berlin. La version française des textes de Kant appartient à l'édition des Euvres philosophiques (trois tomes) chez Gallimard.

${ }^{2} M M, D V$, AA 06: 431. 
la Métaphysique des mours ${ }^{3}$, c'est-à-dire, sur la loquacité, la politesse, l'hospitalité et l'indulgence, une " menue monnaie " $(A p H, \$ 14)$ en comparaison avec la vraie vertu, mais qui font partie de l'effet de trompe d'cil que le sujet pratique doit produire sur sa nature, compte tenu de sa tendance enracinée à l'égoïsme et de la perversion de ses mobiles moraux. Le jeu de la dissimulation, qui oblige les sujets à se présenter comme meilleurs qu'ils ne sont en vérité, impose selon Kant un salutaire obstacle relativement à cette tendance de la nature humaine pour la perversion de la moralité. En deuxième lieu, j'analyserai le lien entre ces "suppléments de la vertu " , qui sont les bonnes manières sociales, et un fait anthropologique incontestable : la faiblesse de la nature humaine et son besoin d'user de béquilles esthétiques pour s'accoutumer à agir suivant des principes pratiques purs. Ces suppléments montrent comment « le goût rend en quelque sorte possible le passage [Übergang] du charme sensible à l'intérêt moral habituel sans un saut trop violent " ${ }^{5}$, et ce beau jeu doit être valorisé pour lui-même parce qu'il nous habitue à une normativité sociale favorable à la fondation d'une communauté éthique et politique. Finalement, j'essayerai de tirer des conclusions sur le besoin de médiations que la nature humaine montre, quand il s'agit d'interagir et de communiquer avec des autres. À mon avis, l'éloge kantien de la politesse doit être compris au-dedans d'une certaine géométrie qui aide l'être humain à se mieux connaître, de sorte qu'on peut vérifier l'affirmation kantienne selon laquelle «seule la descente aux enfers que constitue la connaissance de soi ouvre la voie à l'apothéose». ${ }^{6}$

\section{$* * *$}

\section{Une soumission indirecte à la loi morale : le sens anthropologique de la politesse.}

Le premier pas pour saisir le sens des rituels appartenants à la courtoisie requiert de comprendre celle-ci comme un piège qui va à l'encontre de la source anthropologique des tromperies sur la motivation morale. ${ }^{7}$ Au lieu de recourir à la violence aveugle des inclinations et de l'instinct, la politesse utilise les mêmes moyens que la sensibilité pour bouleverser la scénographie de celle-ci,

${ }^{3} M M, D V, \$ 48$, AA $06: 473$.

4 Observations, AA $02: 217$.

${ }^{5} \mathrm{KprV}$, AA $05: 354$.

${ }^{6} M M, D V, \$ 14, \mathrm{AA} 06: 441$.

7 Vd. Frierson (2005: 108-109). 
qui s'articule à l'amour propre. Kant se montre extrêmement sûr à l'égard de la nécessité d'exécuter cette opération anthropologique:

«[T]romper l'imposteur qui est en nous-mêmes, l'inclination, est, à l'inverse, retour à la soumission à la loi de la vertu, et non point tromperie, mais une manière innocente de nous donner le change». ${ }^{8}$

On choisira des exemples pour commenter ce passage. L'inclination nous présente très habituellement l'inactivité comme la dernière fin physique, la plus souhaitable, mais en vérité il n'y a aucun plaisir plus complet que le repos après le travail ${ }^{9}$, comme s'il y avait quelque chose dans notre nature de radicalement masochiste, qui rejetait toute manifestation absolue du plaisir. Bien que notre esprit croie chercher le loisir sans conditions ${ }^{10}$, le piège déçoit ce trompe-l'œil produit par nos sens, en les trompant, à son tour, dans leur propre illusion. ${ }^{11} \mathrm{Il}$ faut prêter attention au fait que l'illusion $[\text { Schein }]^{12}$ ne doit se confondre pas avec la déception [Betrug] chez Kant. La première serait absolument nécessaire dans la société humaine, bien qu'il faille veiller à éviter la formation de préjugés, selon l'avis de Kant, tandis que la deuxième agace et irrite dans toutes ses manifestations $^{13}$, dans la mesure où elle indique qu'on a chosifié une personne, ce qui est une atteinte à sa dignité. La solidité et la force de l'illusion s'apprécie aussi quand on perçoit, pour proposer ici un exemple, qu'on continue à honorer à un représentant parlementaire bien qu'on soit conscient des activités ludiques ou même ridicules qu'il peut accomplir chez lui, dans son espace privé. Dans tous les cas,

«la vie humaine et ses actions sont un jeu. Telles illusions sont bonnes et rendent la vie agréable, elles plaisent tous et qui veuille les arracher ne fera pas aucun faveur aux êtres humaines». ${ }^{14}$

ApH, $\$ 14$, AA 07: 151.

9 ApH, $\$ 87$, AA $07: 276$.

${ }_{10} \mathrm{ApH}, \$ 14, \mathrm{AA} 07: 152$.

11 Vd. les observations à cet égard de Frierson (2005: 109). Cfr. V-Anth/Fried, AA 25/1: 503.

12 Vd. Kant, ApH, $\$$ 13, AA 07: 149-150 ; cfr. V-Anth/Fried, AA 25/1 : 502-503. Vd. les remarques sur ces passages chez Frierson (2005: 116-117 et 120).

13 V-Anth/Fried, AA 25/1: 505.

14 V-Anth/Fried, AA 25/1: 504. 
Une fois confirmé le fait que tromper les fins que nos sens se forment bien naturellement est un phénomène habituel pour la nature humaine, il semble opportun de légitimer le recours répandu à l'apparence morale, c'est-à-dire d'exposer qu'elle ne représente pas une exception à l'interdit inconditionné de mentir. En ce qui concerne ce doute, la première chose qu'il faudrait déclarer est que l'illusion de la vertu contenue dans la politesse ne devrait pas s'encadrer sous l'opposition entre la vérité et le mensonge — contradictorie oppositis-, mais plutôt sous l'opposition entre la franchise [Offenherzigkeit] et la réserve $[\text { Zurückhaltung }]^{15}$, desquelles on parlera plus avant avec davantage de détails. Kant fait allusion à cette distinction quand il cherche à justifier son comportement devant la censure ${ }^{16}$, parce que la réserve obéit à des limitations de l'esprit humain où l'on trouve toujours un reste d'obscurité irréductible. Seulement la sincérité [Aufrichtigkeit], qui porte à «dire avec véracité tout ce qu'on» serait un véritable devoir pour l'humanité, mais la franchise, une ouverture du cour qui amène à «dire toute la vérité que l'on sait» ${ }^{17}$ ne jouirait pas d'un tel éloge, puisque l'espèce humaine n'est pas préparée à une telle mis en évidence de son intériorité. Notre espèce contient des composants assez dangereux pour la vie en société et un exemple de civilisation consistera à contribuer à les dominer avec ruse. La curieuse lettre que Kant adresse à la malheureuse Maria von Herbert, trop préoccupée par les démons de son intériorité, consiste à justifier cette résistance de l'âme humaine par une explication complète:

"Cette réserve est inhérente aux limites de notre nature et ne corrompt pas à proprement parler le caractère, mais est seulement un mal qui empêche d'en tirer tout le bien possible. Le défaut de sincérité au contraire est une corruption de la manière de penser et quelque chose de foncièrement mauvais». ${ }^{18}$

Même lorsqu'on se trouve «dans le commerce le plus intime avec l'être aimé» (ibid.) une certaine solitude est le destin incontestable de l'être humain. Sur cet obstacle insurmontable dérivé de l'impureté de notre esprit on pourra seulement prendre du recul, déployer des médiations, belles de préférence parce que, tant

$15 M M, D V$, AA $06: 433$, note.

16 Vd. Lettre à Mendelssohn du 8 Avril de 1766, AA 10 : 69: «Sans doute je pense avec la conviction la plus claire, et pour ma plus grande satisfaction, beaucoup de choses que je n'aurais jamais le courage de dire, mais jamais cependant je ne dirai quelque chose que je ne pense point».

17 Rel., AA 06: 190, n.

$18 \mathrm{Br}$, AA $11: 332$. 
que nous restons des hommes, il n'est pas possible de produire une conduite capable de supprimer l'opacité surgie dans l'interaction entre les sujets. ${ }^{19}$ Prononcer un contenu manifestement mensonger n'a rien à voir avec une déclaration incomplète, qui laisse des informations légitimement en réserve. S’il est vrai qu'on ne peut tirer de l'espèce humaine tout le bien possible, on maintiendra l'espoir de son rapprochement progressif avec un état de moralisation, progrès qui se produira avec une conscience lucide des failles auxquelles remédier. Les observations antérieures dessinent un portrait de la condition humaine qui suppose la capacité d'origine culturelle de ne pas se laisser tromper par les différentes figures de la galanterie sociale :

«Les révérences (compliments) et tout l'attirail de galanterie de cour ainsi que les plus chaudes protestations d'amitié ne sont certes pas toujours précisément la vérité $[\ldots]$, du moins ne trompent-ils pas pour autant, parce que chacun sait à quoi s'en tenir, et surtout, vides tout d'abord, mènent peu à peu à de réelles dispositions de cette sorte». ${ }^{20}$

Dès que nous somme éduqués et parcourons un procès de civilisation, nous sommes aussi préparés à interpréter, à l'instar d'êtres mûrs, sans tomber dans l'erreur, le sens purement formel de la bonne conduite. L'illusion de la vertu contenue dans tout compliment lié au simple compromis, par exemple, expliquerait le phénomène psychologico-social qui fait que l'honorabilité [Ehrbarkeit], obtenue par la conduite courtoise d'un homme, puisse être généralement interprétée comme le produit d'une véritable intention morale. ${ }^{21}$ La virtualité qui consiste à produire un intérêt pour la vie morale, quoique ce dernier soit mobilisé par l'attrait de la valorisation sociale, suffirait à juger comme étant une grande erreur la considération de l'apparence morale comme un mensonge méprisable :

«Toute vertu humaine du domaine des relations n'est que vile monnaie: quiconque la prend pour or véritable est un enfant. Mieux vaut pourtant avoir

19 P. Tunhas a prêté attention à cette question avec une lucidité remarquable (2003: 221222) : «Toutes les fois qu'il y a une certaine latitude dans notre champ de réponses — toutes les fois où l'on n'est pas obligé de dire oui ou non —il y a lieu pour la réserve. Il est vrai qu'elle sera toujours indice d'un abîme entre les êtres, mais elle ne corrompt pas notre caractère : elle signale simplement ses limites. Et ces limites sont celles qui lui sont inspirés par la prudence».

${ }^{20}$ ApH, \$ 14, AA 07: 152.

21 V-Anth/Mensch, AA 25/2: 929. 
en circulation une vile monnaie que nulle espèce de cet aloi ; elle peut à la fin, bien qu'avec une déperdition considérable, se convertir en or pur». ${ }^{22}$

Prenant en compte les observations comme celle faite antérieurement, Kant devrait être accueilli comme un des pères de la théorie de la culture, une discipline si chère aux néo-kantiens comme E. Cassirer ${ }^{23}$, dès lors qu'il essaie de découvrir, entre autres enjeux relatifs à la Menschkenntnis, les principes anthropologiques responsables de la tendance de l'espèce humaine à la comédie. Comme d'habitude en anthropologie, il s'agit de procéder selon une méthode analytique, c'est-à-dire qu'il faut partir des jeux sociaux pour reconnaître quelles sont leurs conditions constitutives et trouver ce qui leur permet de suivre leur cours sans entraves:

«Les hommes sont dans l'ensemble d'autant plus comédiens qu'ils sont plus civilisés : ils adoptent l'apparence de l'affection, du respect d'autrui, de la décence, du désintéressement, sans tromper personne, car chacun parmi les autres entend bien que le cœur n'y a point de part ; il est au demeurant très bon qu'il en aille ainsi dans le monde. Par le fait que des hommes jouent ces rôles, les vertus qu'ils se sont, un certain temps, contentés d'affecter, finissent bien par être éveillés, et elles passent dans leur disposition d'esprit». ${ }^{24}$

L'être humain se conduit comme un comédien par rapport aux autres parce qu'il sent une inclination à vivre en société, mais cette tendance est dotée d'une certaine complexité, dès lors que la simple coexistence avec les autres êtres humains représente aussi le surgissement du mal et des passions regrettables. Dans un texte remarquable de l'écrit sur la religion, Kant soutient qu'il est impossible de se trouver entouré par d'autres hommes sans commencer à se comparer à eux et à leurs propriétés et capacités, avec pour conséquence d'éprouver le regret ou l'orgueil qui procèdent de cette comparaison :

${ }^{22}$ ApH, $\$ 14$, AA 07: 152. Vd. Frierson (2005: 111).

23 On pourrait citer l'Essai sur l'homme de Cassirer (Paris, Minuit, 1977), sans oublier la proximité entre l'analyse fragmentaire des moyens du processus de civilisation chez Kant et l'œuvre désormais classique de N. Elias sur ce sujet (La civilisation des moeurs, Paris, Pocket Agora, 1973).

${ }^{24} \mathrm{ApH}, \$ 14, \mathrm{AA} 07: 151$. 
"L'envie, la soif de domination, la cupidité et les inclinations haineuses qui s'y relient troublent aussitôt sa nature, en elle-même suffisante, lorsquỉl se trouve entre les hommes. [...] [I]l suffit qu'ils soient là, qu'ils l'entourent, que ce soient des hommes, pour qu'ils se corrompent réciproquement dans leur disposition morale et se rendent mauvais les uns aux autres» (Rel., AA 06: 93-94 ; OP, III : 113).

Les passions se répandent rapidement là où les hommes coexistent, de sorte qu'il faut imposer des obstacles à leur cours, si on ne veut pas que la sociabilité devienne un champ de destruction. Il faudra rechercher la voie intermédiaire représentée par l'insociable sociabilité qui, contrairement à ce qu'on considère quelques fois, n'est pas une donnée naturelle, mais un produit du développement culturel. La distinction entre la réticence ou la réserve [Zurückhaltung], couchée sous la dissimulatio latine, et la simulation [Verstellung; simulatio] s'explique par le fait que notre nature est trop chargée d'iniquités pour avoir la licence de nous présenter tels que nous sommes aux autres. ${ }^{25}$ La haine et le mépris réciproques résulteraient d'une telle manifestation complètement ouverte de l'intériorité des cœurs humains. C'est cette situation qui explique que la beauté, la grâce, si chères à Schiller, doivent préparer son chemin à la vertu. ${ }^{26} \mathrm{La}$ dissimulation nous aide à masquer nos propres défauts, lisait-on dans la lettre de Kant à Maria von Herbert. La réserve nous permet ainsi de maintenir en latence les traits plus honteux et insatisfaisants de notre esprit, une conduite très correcte si l'on veut conserver l'espoir de remédier à la déficience de traits plus adéquats à notre destination morale.

Dans le contexte d'une évaluation si positive de l'art de la dissimulation, il est cependant essentiel de rappeler que la seule apparence qui doive être effacée du monde humaine est l'apparence du bien en nous-mêmes :

${ }^{25} \mathrm{Vd}$. un très éclairant texte sur ce sujet dans V-Mo/Collins, AA 27 : 444). Cfr. la lecture que Mahon (2003 : 117s.) fait de cette distinction kantienne.

${ }^{26}$ C'est bien connue la condamne kantienne de la compréhension de l'ordre entre grâce et dignité chez Schiller, vd. Rel., AA 06: 23, note: «[C]omme il n'est question encore que de la morale, [les Muses] se tiennent à distance respectueuse. Si l'on envisage les conséquences gracieuses que la vertu répandrait dans le monde, si elle pouvait pénétrer partout, alors, en ce cas, la raison moralement dirigée (par le truchement de l'imagination)» met en jeu la sensibilité. Hercule ne devient Musagète qu'après avoir dominé les monstres, travaux devant lesquels ces bonnes sœurs reculent. Les compagnes de Venus Uranie sont dans la suite de la Venus Dionée des courtisanes dès qu'elles s'immiscent dans le travail consistant à déterminer le devoir et veulent livres les motifs appropriés». 
«Seule l'apparence du bien en nous-mêmes doit être effacée sans ménagements, comme doit être déchiré le voile dont l'amour-propre recouvre nos faiblesses morales; car l'apparence exerce sa tromperie là où l'on fait miroiter à ses propres yeux, à l'aide d'images dénuées de tout contenu moral, l'effacement de la faute ou, par la récusation de celle-là, la conviction de n'être en rien coupable, par exemple quand, à la fin de la vie, on dépeint le repentir des mauvaises actions sous les couleurs d'un amendement véritable, ou une infraction délibérée sous celles d'une faiblesse humaine». ${ }^{27}$

L'apparence morale produite par le sujet par rapport à lui-même sera interdite sans ambages, parce que chacun doit éviter de tomber dans de fausses illusions sur la pureté de ses intentions. C'est le seul champ où la dissimulation n'est pas bienvenue, parce que les fantômes de la justification frauduleuse de soi hantent spécialement le dialogue intérieur que le sujet entame avec lui-même. ${ }^{28} \mathrm{Il}$ faut assumer que même l'examen le plus rigoureux pourrait pénétrer jusqu'aux mobiles secrets de nos actes :

«S'être donné pour maxime suprême la véracité dans l'intériorité de l'aveu à soi-même et en même temps dans la conduite à l'égard de tout autre, est l'unique preuve de la conscience qu'un homme peut avoir de posséder un caractère»..$^{29}$

Dans une telle situation le seul chemin qui reste ouvert sera un constant contrôle des identités imaginaires que chacun s'attribue à lui-même, en se servant principalement de l'interrogation constamment renouvelée sur sa propre Gewissenhaftigkeit, qualifiée par F. Calori de «d'inquiétude morale salutaire» ${ }^{30}$ et par P. Tunhas de «solitude intérieure à la solitude» ${ }^{31}$, propres à une intériorité qui ne se laisse pas séduire par les promesses d'une sainteté irréalisable par l'être humain

${ }_{27}$ ApH, $\$ 14$, AA 07: 153.

${ }^{28}$ Vd. les précieux remarques de Calori sur ce sujet (2011: 18) : «L'impossibilité de pénétrer les profondeurs du cœur humain et d'acquérir une certitude quant à la valeur morale de notre maxime fondamentale n'annule cependant pas l'impératif de connaissance de soi : elle interdit seulement que nous puissions acquérir sur ce point une quelconque certitude».

29 ApH, AA 07: 295.

30 Calori (2011: 19).

31 Tunhas (2003: 227). 
et qui en même temps ne tombe pas en proie au désespoir relativement à sa capacité d'améliorer son état moral, c'est-à-dire qui se garde bien des dangers de l'auto-mortification. Le formalisme éthique kantien avec ses remarques montre qu'il sait très bien que l'amour-propre produit le fait que l'homme reste toujours un mystère pour lui-même, qu'il est un être qui se trahit lui-même, et tout spécialement la partie de lui-même qu'il considère consciemment comme la plus précieuse — video meliora proboque, deteriora sequo. Tout homme est un traitre de soi, comme le prouve en outre l'ambivalence et la variabilité de notre concept de bonheur. Mais ce pessimisme moral contrôlé ne doit pas nous conduire à perdre notre temps en identifiant sans relais les traces de l'Unlauterkeit dans chacune de nos quotidiennes. ${ }^{32}$ Les exigences de dissimulation qui résultent des convenances sociales trompent les motifs reliés à l'amour-propre et accoutument l'être humain à adopter des fins qui ne l'intéressent pas directement comme un être sensible. Se montrer apparemment intéressé par des buts qui pouvaient d'abord laisser notre esprit indifférent nous rend progressivement accoutumé à les choisir spontanément, quoiqu'il ne puisse y avoir une continuité entre ces deux états.

\section{L'esthétisation du monde et les parerga de la vertu morale.}

Bien qu'ils ne puissent disposer d'une véritable systématicité, plusieurs passages kantiens se montrent confiants dans la capacité des apparences générées par la politesse humaine à favoriser l'adoption d'un vrai mode de pensée moral, comme si la nature humaine conduisait à se tromper soi-même pour empêcher que l'amour-propre ne croisse démesurément. Les règles pragmatiques de la bonne conduite pousseront les sujets à adopter la vertu comme un moteur intérieur, de sorte que les expressions de la politesse deviendront des parerga de la vertu morale, comme le déclare un passage de la Doctrine de la vertu:

"Certes, ce ne sont là que des dehors ou accessoires (parerga) qui donnent une belle apparence de vertu, mais une apparence qui n'est pas trompeuse parce que chacun sait quel cas il doit en faire. Ils ne comptent il est vrai que pour de la menue monnaie, mais renforcent pourtant le sentiment de la vertu lui-même par l'effort accompli pour rapprocher autant que possible cette apparence de la vérité, effort de se laisser facilement aborder, effort de loquacité, de politesse, d'hospitalité, d'indulgence (éviter la querelle dans la

${ }^{32}$ Vd. $M M, D V$, AA $06: 409$. Cfr. Tunhas (2003: 228-229). 
controverse), tout ceci n'étant que les bonnes manières du commerce humain assorties des obligations extérieures par lesquelles on oblige en même temps les autres, qui contribuent donc bien à l'intention vertueuse, dans la mesure où elles rendent la vertu au moins aimable».

La vertu rejette tous les exemples, comme Kant ne manque jamais de le rappeler, mais la conduite polie et sociable, à l'instar d'une sorte de simulacre ${ }^{33}$ de la vertu, reçoit la licence de se situer discrètement à côté de celle-ci dans l'anthropologie morale. La liste des vertus homilétiques, c'est-à-dire des vertus appartenant au développement d'un idéal de sociabilité, contribue à créer une ambiance pour ainsi dire harmonieuse avec les propos de la vertu morale. En réalité, sur ce point, Kant n'invente rien ${ }^{34}$, parce que l'Ethica de A. Baumgarten contenait dans sa troisième partie (\$\$378-386) des observations intéressantes sur les officia erga alia, devoirs où se démarquent par leur importance les officia conversationis, qui ne doivent pas abuser de l'esprit contradicteur ou de l'amour pour les paradoxes, mais chercher à se guider par le sens commun partagé par la plupart des hommes. Quelques cours de morale de Kant recueillent des commentaires précieux sur l'«exercice et la culture de la vertu» [Übung und Cultur der Tugend], où on trouve défendue l'idée que la courtoisie et la politesse contribuent à faire de l'inclination animale l'objet d'une inclination plus raffinée. ${ }^{35}$ Vivre isolé éloigne de la vertu dans la mesure où cette forme de vie nous installe dans l'égoïsme, tandis qu'une vie conduite dans une aimable société avec les autres constitue un devoir late dictus, c'est-à-dire un devoir de vertu. ${ }^{36}$

${ }^{33}$ J'emprunte cette remarque à l'article de R. Rodríguez Aramayo (2013), spécialement la section «Del simulacro como método hacia la virtud». Vd. aussi la complète monographie que $\mathrm{O}$. Custer $(2012: 341)$ a consacrée à l'étude de l'exemple dans la philosophie morale de Kant : «Si les accessoires de la vertu peuvent conduire ailleurs qu'à l'hypocrisie c'est parce qu'elles peuvent conduire à un "faire»; un Zutat peut mener à un Tat. Voilà comment le texte kantien fournit une argumentation positive en faveur de la possibilité de la liberté. La liberté doit se faire, comme un œuvre ; elle peut se faire par une imitation exemplaire à la manière du génie qui s’inspire de ses prédécesseurs".

34 J. Stohr (2010 : 7ss. et 17-19) s'est servi de la théorie sociologique de E. Gofman (The Presentation of the Self in Every Day Life, New York, Anchor Books, 1959) pour reconnaitre dans le discours kantien sur la dissimulation et la politesse un «moral front» qui aide à adopter des véritables intentions morales dans l'action humaine. Vd. Driver (1992) passim.

35 Vd. V-Mo/Collins, AA 27: 456.

${ }^{36} M M, D V \$$ 48, AA 06: 473: «C'est un devoir aussi bien envers soi-même qu'envers les autres de se livrer au commerce que les hommes ont entre eux (officium commercii, sociabilitas), de ne pas s'isoler (separatistam agere); de faire de soi assurément le point central 
Dans tous les cas, les avantages qui jusqu'ici ont caractérisé l'art de dissimuler ses propres pensées et désirs en société ne peuvent pas faire oublier l'ambiguïté inhérente à la tendance de l'être humain à déguiser ses propres pensées et à découvrir celles de l'autre. Les pages finales de la Caractéristique de l'Anthropologie, publiée en 1798, montrent clairement qu'en prenant en compte les traits que la dissimulation a donné à notre espèce, il faudrait considérer cetteci comme une caricature de soi-même, qui aurait profondément satisfait des auteurs comme Baudelaire, puisque la constitution anthropologique humaine amène assez facilement jusqu’à la mystification et finalement au mensonge. Ce qui donne une idée juste de la véritable fonction de cet esprit de déguisement de soi est le fait que les êtres humains réprouvent intérieurement très fortement cette tendance, une réaction qui permet le représenter «comme un genre d'êtres raisonnables tout à l'effort de se hisser du mal vers le bien en un progrès continu, au milieu d'obstacles». ${ }^{37} \mathrm{La}$ "contrainte de l'éducation» [Zwang der Anständigkeit ${ }^{38}$ que les hommes engagent opère avec une effectivité inusitée, si on considère le fait qu'il s'agit d'un dispositif esthétique, qui conduit les hommes à se juger réciproquement selon qu'ils respectent ou pas les règles plus implicites qu'explicites des modes sociales. Un respect esthétique annonce l'événement d'un respect plus intérieur, qui concerne le rapport propre à la loi. En effet, la capacité d'adopter une conduite apparemment morale révèle un «maitrise de soi» [Selbstbeherrschung] et une "contrainte de soi» [Selbstbezwingung] qui témoignent de l'accomplissement de la destination morale de notre espèce. ${ }^{39}$ Pour cette raison, ces dispositions, liées à la fabrique d'illusions enracinée dans la nature humaine, sont considérées comme le "premier pas vers la vertu» et même comme un «analogon ${ }^{40}$ du fondement de la morale. Kant argumente en faveur d'un recours commun aux effets bienfaisants de l'imitation, de sorte que toute médisance soit bannie de la scène sociale, comme l'indique la Doctrine de la vertu:

"C'est donc un devoir de vertu que de jeter le voile de la philanthropie sur les fautes des autres, non seulement en adoucissant nos jugements,

\footnotetext{
immuable de ses principes, mais aussi pourtant de considérer ce cercle tracé autour de soi comme une partie d'un cercle embrassant tout, celui de l'intention cosmopolitique, non pas précisément pour travailler au bien du monde comme à une fin, mais uniquement pour cultiver les moyens qui y conduisent indirectement, l'aménité en société, l'esprit de conciliation, l'amour et le respect réciproques (l'affabilité et la bienséance, humanitas aesthetica et decorum) et d'ajouter ainsi les grâces à la vertu — ce que faire est même un devoir de vertu».

37 ApH, AA $07: 332$.

38 V-Anth/Fried, AA 25/1: 692.

$39 V$-Anth/Mensch, AA 25/2: 930. Vd. Frierson (2005: 112).

40 -Anth/Mensch, AA 25/2: 928.
} 
mais aussi en les passant sous silence : car les exemples de respect que nous donnons aux autres peuvent aussi susciter en eux l'effort pour le mériter également». ${ }^{41}$

Les amis devront se communiquer les uns aux autres leurs défauts ${ }^{42}$, pour autant qu'il n'en résulte pas une situation trop pénible pour leur amitié. La rencontre avec un ami est décrite par Kant comme la sortie rêvée d'une prison, de sorte que le sujet ne se sent plus isolé avec ses propres pensées, et jouit d'une liberté qu'il ne peut avoir dans la masse. ${ }^{43}$ Mais les devoirs de l'amitié ne s'étendent pas sur toute la surface de la Terre. Il serait donc préférable de ne pas répandre sans scrupules les échecs malheureusement assez partagés des sujets qui doivent atteindre leur état de moralisation et faire comme si nous étions meilleurs que ce que nous sommes dans la vie réelle, c'est-à-dire agir comme des comédiens vertueux. La curiosité pour les défauts d'autrui pervertit la disposition humaine au bien. Peut-être est-ce pour cette raison que Kant remarque, dans ses cours d'éthique, avec un ton qui ne lui est pas coutumier, que bien que nous ayons tous chez nous une toilette, nous ne la montrons pas aux invités parce qu’on considère que cette conduite n'est pas de bon goût. Il va plus loin par rapport à ce «voile de philanthropie», qui se révèle hautement utile pour le développement de la nature humaine, quand il affirme qu'il faut cultiver les sentiments qui mettent à l'épreuve notre sens de l'humanité.

Tout un groupe de vertus seront convoquées effectivement dans ce contexte de discussion. La sympathia moralis, par exemple, qui manifeste un état de plaisir et déplaisir par rapport au contentement et au mécontentement des autres êtres humains, signifie pour Kant un devoir d'humanité (MM, $\$ 34$, AA $06: 456$ ). Il est bien naturellement préférable que cette sensibilité pour les sentiments d'autrui se manifeste comme une humanitas practica plutôt que comme une humanitas aesthetica, parce que la seconde n'est pas libre (ibíd.), c'est-à-dire ne consiste pas dans la faculté de communiquer ses propres sentiments, mais dans une séquence mécanique et corporelle qui rend possible une transmission sentimentale. L'humanité définie dans le $\$ 60$ de la Critique du jugement comme un sentiment de participation universelle et comme une faculté de se communiquer dans le mode plus universel et intime ${ }^{44}$, qui combinés délimitent la sociabilité

\footnotetext{
${ }^{41} M M, D V, \$ 43, \mathrm{AA} 06: 466$.

${ }^{42} M M, D V, \$ 46, \mathrm{AA} 06: 470$.

$43 M M, D V$, AA $06: 471-472$.

${ }^{44} K U, \$ 60$, AA $05: 355$.
} 
humaine, se voit exercée seulement dans l'humanitas practica. ${ }^{45}$ L'exercice de la sympathie pour le mode de sentir des autres appartient à un ordre du sens qui ne possède pas une signification déterminée pour les êtres humains, comme il arrive quand on écoute en société un mot d'esprit qui provoque le rire des participants dans une conversation, un avènement qui permet que la coexistence conviviale continue et qui couronne une soirée passée dans la Tischgesellschaft. De quoi est-ce qu'on rit, quand on écoute quelque chose de drôle?. En vérité, on rit parce que le rien, cet énigmatique contenu de toute table analytique des concepts métaphysiques, advient à la présence ${ }^{46}$ et cette position vide remplit une fonction essentielle dans la connaissance de l'être humain et de ses capacités comme de ses limites.

\section{La géométrie blanche des bonnes manières.}

On ne rencontrera pas un texte de Kant plus éclairant sur le besoin que l'espèce humaine a de l'art de la dissimulation, qu'une hypothèse utilisée dans les dernières pages de la section concernant les traits descriptifs du caractère de l'humanité dans l'Anthropologie d'un point de vue pragmatique. Dans ce texte bien connu, on remarque que les êtres humains ne pourraient pas continuer à coexister s'ils ne pourraient "penser qu'a haute voix [...], incapables d'avoir, dans la veille ou en rêve, en société ou seul, des pensées qu'ils n'exprimeraient pas aussitôt». ${ }^{47}$ Comme on l'a vu un peu auparavant, notre nature se trouve en constant effort pour sortir d'un état de détresse relatif à l'atteinte de ses buts moraux, de sorte qu'adopter une franchise absolue, sans préjugés, au sein de la communauté humaine, en imitant la transparence pratiquée par les êtres raisonnables qui habitent d'autres planètes, supprimerait toute possibilité de coexistence avec eux, en empêchant même qu'ils puissent se respecter ou arriver

45 Cfr. l'analyse de F. Trentani sur l'importance de cultiver ces dispositions sentimentales en bénéfice de la propre morale (2013: 95s.). Cfr. Cagle (2005 : 459) sur la convenance de connaittre le mode de sentir d'autres personnes comme le trait d'un caractère moral mature.

${ }^{46} \mathrm{Vd}$. la définition du rire par Kant dans la troisième Critique comme la transformation subite d'une longue attente en rien ( KU, AA $05: 332$; cfr. $A p H, \$ 88$, AA $07: 281)$. Vd. Tunhas (2003 : 225) : «Donc, la dissimulation et l'art de l'apparence appartiennent à un domaine que l'on pourrait appeler neutre, qui ne joue jamais un rôle principal dans les différends (les vraies disputes sont celles du caractère intelligible), et qui reste subordonné à la moralité». Sur le rire chez Kant et son rapport avec l'esprit de convivialité vd. Tagliapietra (2012: 134s.).

${ }^{4}$ ApH, AA $07: 332$. 
au moindre accord. ${ }^{48}$ Une trace de cette opacité foncière des êtres humains restera dans la même fondation kantienne de la forme de la publicité, qu'il serait gravement erroné de réduire à la transparence. ${ }^{49} \mathrm{Il}$ est sûr qu'une maxime qui ne peut pas s'exprimer à voix haute sans courir le risque d'échouer doit être considérée sans ambages comme injuste ${ }^{50}$, mais un impératif de transparence aurait le redoutable avantage de rappeler l'obsession de tous les régimes totalitaires cherchant à envahir l'intériorité et la vie privée des citoyens. Les savants des Lumières essayent de convaincre les seigneurs du monde qu'ils devraient s'intéresser, même à leur profit personnel de gouvernants, à la garantie de la liberté d'expression de leurs citoyens, le palladion des peuples de la Terre. Mais avoir la garantie de pouvoir s'exprimer sans censure d'aucun type ne coïncide pas avec une parrhesie sans limites. On ne peut pas négliger le fait que le célèbre motto de Qu'est-ce que sorienter dans la pensée — - "penserions-nous beaucoup et penserions-nous bien si nous ne pensions pas pour ainsi dire en commun avec d'autres» (AA VIII : 144) — ne commande pas de communiquer sans limites nos pensées aux autres, mais soutient que cette communicabilité doit être garantie par le législateur, si on ne veut pas que la pensée humaine s'atrophie. Tout change dans la lecture de ce texte si la possibilité de se communiquer devient la nécessité d'avouer. ${ }^{51} \mathrm{Kant}$ ne cache pas le fait que certains gouvernements, comme la constitution de la monarchie britannique de laquelle il parle dans Le conflit des facultés, feignent d'être guidés par l'esprit de la publicité, qui se laisse donc pervertir. Parfois on juge très hâtivement le fait que la simple apparence de discussion publique est préférable par rapport aux gouverneurs qui en censurant les discours qu'ils présentent à leur peuple ${ }^{52}$, en exerçant cet art qu'aujourd'hui on appelle marketing, rendent un hommage inattendu au droit. Bien au contraire, le roi britannique laisse les Assemblées discuter de tout ce qu'elles veulent, en les maintenant dans un permanent état de vie parlementaire fictive, dans la mesure où il garde le

48 Le meilleur travail sur l'usage que Kant fait du point de comparaison représenté par le alien est de D. Clark (2001 : 248ss.). Vd. aussi Cohen (2008 : 87-88) : «Kant does not in fact reject Rousseau's claim that opacity is a humane evil ; rather, he makes a complementary and equally valid point, namely that opacity is at once an expression of the evil in human nature and part of the necessary remedy for it. For what appeared as a moral flaw of the human species turns out to prevent it from self-destruction and allow peaceful relations amongst its members».

49 Sur cette différence le lecteur pourra consulter avec sûr profit le travail de F. Calori, « «Laut denken»: de la transparence chez Kant», Raison publique (2011) passim.

50 PP, AA 08: 381.

${ }^{51}$ Vd. Calori (2011: 4): «Le texte ne dit pas que toute maxime politique doit être proclamée publiquement, mais seulement qu'elle doit pouvoir l'être».

52 Vd. PP, AA 08: 355. 
monopole du droit de déclarer la guerre ${ }^{53}$, privilège propre au monarque absolu, privilège arraché en conséquence au peuple.

C'est l'attention au lestage que le mal radical laisse dans la nature humaine qui conduit Kant à soutenir que les jeunes doivent apprendre à «se rendre impénétrable[s]», sans négliger l'art de pénétrer les pensées d'autrui ${ }^{54}$, une sentence qui garde une position d'excellence dans l'Oracle manuel du jésuite espagnol Baltasar Gracián. Dans l'édition Rink du cours de Kant sur la pédagogie, on lit que c'est l'impureté [Unlauterkeit] qui est le trait responsable de l'admission de la dissimulation comme recommandable pour la formation des enfants qui devront agir sur le monde. ${ }^{55}$ Le monde sera donc meilleur si l'on jette un voile sur les failles les plus honteuses de l'espèce humaine, le «voile de la philanthropie», afin d'obtenir que l'extension de la bonne conduite dans toutes les couches sociales provoque une saine mimesis, toujours bienfaisante pour le progrès de la vertu. Sans arriver aux positions extrêmes de Diderot dans Le paradoxe sur le comédien, Kant déclare que l'imitation servira, particulièrement à l'homme encore inculte, comme "première détermination de la volonté à admettre des maximes qu'il fait siennes par la suite». ${ }^{56}$ Bien évidemment, la spécificité de l'Exempel par contraste avec le Beispiel des sciences théoriques, concerne l'identification de la faisabilité ou practicabilité57 [Tunlichkeit] de l'action morale. Selon les principes de l'anthropologie morale kantienne, on reconnaitra que la médisance doit être bannie du monde. En effet, «les exemples de respect que nous donnons aux autres» (MM, AA $06: 466$ ) encouragent l'effort qu'ils entreprendraient pour mener une vie vertueuse, leur permettant de mériter aussi l'appréciation publique. Qu'estce que la politesse produit donc dans le champ anthropologique? La réponse la plus juste à cette question serait l'établissement de distances entre les sujets, un sujet essentiel dans la théorie pédagogique de Rousseau, dont Kant s’inspire fortement. En réalité, les conséquences de la politesse vont chez Kant bien audelà de la sphère éthique. Même le droit cosmopolitique introduit la garantie d'un droit de visite universel pour tous les êtres humains, dès lors que tous ont un droit de possession commune de la Terre, sans toutefois que cette hospitalité

53 Vd. CF, AA 07: 90.

${ }^{54}$ Vd. Päd., AA 09: 486: «[I]1 lui faut dissimuler et se rendre impénétrable, mais savoir pénétrer autrui. Il lui faut surtout pratiquer la dissimulation pour son caractère. L'art de l'apparence est la convenance [Anstand]. Cet art, il importe de le posséder. Pénétrer les autres est difficile, mais on doit de toute nécessité entendre cet art pour se rendre soi-même impénétrable».

55 Vd. Calori (2011: 8). Cfr. Tunhas (2003: 223-224).

${ }^{56} M M, D V, \$ 52$, AA $06: 479$. Vd. Custer (2012: 232ss.).

${ }^{57} M M, D V, \$ 52$, AA $06: 479$. 
reçoive une portée uniquement morale ou philanthropique, puisqu' elle est un véritable principe juridique. ${ }^{58}$ Les effets que la galanterie produits dans le rapport entre les sexes seront suffisamment éloquents pour cette ouverture de distances produite par la politesse. Les règles de la grammaire sociale (conversation galante, éloges...) appliquée à l'ordre sexuel apaisent progressivement la violente force de la sexualité et commencent à gouverner tout contact entre homme et femme, en remplaçant la domination de l'instinct :

« $[\mathrm{L}] \mathrm{a}$ décence (pudicitia), contrainte exercée sur soi-même, qui dissimule la passion, est une illusion fort salutaire pour susciter entre l'un et l'autre sexes la distance nécessaire à ce que l'un d'eux ne soit pas avili en simple instrument de jouissance de l'autre. En tout étant de cause, tout ce que l'on désigne du nom de convenances (decorum) est du même genre, à savoir rien d'autre que belle apparences. ${ }^{59}$

Le sentiment de la décence est une sorte de prison produite par le désir humain, qui ainsi remporte paradoxalement sa propre perpétuité. L'histoire conjecturale exposée par Kant fait de la dissimulation une manifestation étonnante de la raison, qui par le moyen de la décence [Sittsamkeit] impose des entraves à "ce qui pourrait éveiller du mépris» ${ }^{60}$ dans la communauté humaine, en imprimant ainsi un changement progressif du mode de penser de l'être humain, qui ouvre la voie à un progrès culturel soumis à la moralité. Dans tout cas, le rapport entre les moyens civilisateurs et la moralité pourrait être exprimé comme une dialectique négative, parce que touts les ponts et passerelles qui produiraient une continuité entre ces deux manifestations de la raison sont destinés à échouer chez Kant. Si l'anthropologie d'un point de vue pragmatique, et non scolastique, pouvait être regardée, ainsi que Kant l'affirme dans les cours universitaires consacrés à cette matière, comme une sorte de géométrie spatiale par rapport à la géodésie ${ }^{61}$, on devrait reconnaître que l'art de la dissimulation contribue à aider les êtres humains à se déplacer sur un fonds obscur qui concerne leur auto-connaissance morale et qui ne fait pas de grands progrès. En fin de compte la courtoisie surgit pour permettre à l'homme d'apprendre à s'accepter avec ses défauts et ses fragilités. Se considérer seulement comme un être rationnel fini

58 Vd. PP, AA 08: 357-358; cfr. $M M, D D, \$ 62$.

$59 \mathrm{ApH}, \mathbb{1}$ 14, AA 07: 152.

60 Conjectures..., AA 08: 113.

${ }^{61}$ V-Anth/Mron, AA 25/2: 1211. Sur l'effet débilitant que la politesse produit par rapport à les inclinations vd. Starobinski (1989) passim et Tagliapietra (2012: 120). 
signifierait survoler de manière injustifiée une entière dimension de soi-même, c'est-à-dire celle du "cher moi», qui ouvre à l'égoïsme dans la conduite, mais qui en même temps offre toujours des nombreuses occasions à la morale de prendre sa juste revanche et de l'emporter dans le champ des mobiles du désir humain.

\section{Bibliographie secondaire}

Cagle, R. "Becoming a Virtuous Agent: Kant and the Cultivation of Feelings and Emotions", Kant-Studien 96/4 (2005), pp. 452-467.

Calori, F. "Laut Denken”: de la transparence chez Kant», Raison publique (2011) : http://www.raison-publique.fr/article $448 . \mathrm{html}$

Clarke, D. «Kant's aliens. The Anthropology and its others», The New Centennial Review 1 (2001), pp. 201-289.

Cohen, A.A. «Kant's Concept of Freedom and the Human Sciences », Canadian Journal of Philosophy, vol. 39/1 (2009), pp. 113-135.

— Kant on Anthropology and Alienology. The Opacity of Human Motivations and Its Anthropological Implications», Kantian Review, vol. 13/2 (2008), pp. 85-106.

Custer, O. L'exemple de Kant, Louvain, De Peeters, 2012.

Driver, J. «Caesar's Wife: On the Moral Significance of Appearing Good», Journal of Philosophy 89, no. 7 (1992): 331-343.

Fenves, P. Late Kant. Toward another Law on the Earth, New York/London, Routledge, 2003.

Frierson, P. Freedom and Anthropology in Kant's Moral Philosophy, New York, CUP, 2003.

— The Moral Importance of Politeness in Kant's Moral Anthropology», Kantian Review, vol. 9/1 (2005), pp. 105-127.

GuYER, P. Kant and the Experience of Freedom: Essays in Aesthetics and Morals, New York, CUP, 1993.

Mahon, J.E. «Kant on Lies, Candour and Reticence», Kantian Review 7 (2003), pp. 102-133.

Munzel, F.G. Kant's Conception of Moral Character: The Critical 'Link' of Morality, Anthropology and Reflective Judgment, Chicago, Chicago U.P., 1999.

Rodríguez Aramayo, R. «La política y su devenir histórico en el pensamiento de Kant», Ideas y Valores (2013), pp. 15-36. 
DOs SANTOS, R. «Virtude, realização da moral e dever de amor aos seres humanos em Kant», Intuitio 5/1 (2012), pp. 4-18.

Starobinski, J. Le remède dans le mal. Critique et légitimation de l'artifice à l'âge des Lumières, Paris, Gallimard, 1989.

SToнr, K. «Keeping the shutters closed: reserve and intimacy on social life» (2010), accessible en: http://northamericankantsociety.onefireplace.org/papers.

TAgliapietra, A. «Tra corpo e spirito. Kant e l'abbozzo di un'antropologia della conversazione», I castelli di Yale 12 (2012), pp. 115-146.

Trentani, F. «La concretezza dell'esperienza morale nella filosofia pratica di Kant», Estudos Kantianos, I/1 (2013), pp. 81-104.

Tunhas, P. «Intention, bonheur et dissimulation», en : M. Cohen-Halimi (coord.), Kant. La rationalité pratique, Paris, PUF, 2003, pp. 173-232.

Wood, A. Kant's Ethical Thought, New York, CUP, 1999.

Recibido: 8/04/2014

Aceptado: 20/10/2014

Este trabajo se encuentra bajo una licencia de Creative Commons ReconocimientoNoComercial-SinObraDerivada 4.0

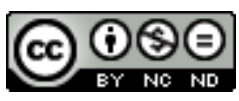

